\title{
Effect of Different Volumes of Interval Training and Continuous Exercise on Interleukin-22 in Adults with Metabolic Syndrome: A Randomized Trial
}

This article was published in the following Dove Press journal: Diabetes, Metabolic Syndrome and Obesity: Targets and Therapy

\author{
Joyce S Ramos ${ }^{1,2}$ \\ Lance C Dalleck ${ }^{2,3}$ \\ Rebecca C Stennett ${ }^{\prime}$ \\ Gregore I Mielke (i)' \\ Shelley E Keating (D) \\ Lydia Murray ${ }^{4}$ \\ Sumaira Z Hasnain ${ }^{4,5}$ \\ Robert G Fassett ${ }^{1}$ \\ Michael McGuckin ${ }^{4,6}$ \\ Ilaria Croci (iD) ${ }^{1,7}$ \\ Jeff S Coombes'
}

'Centre for Research on Exercise, Physical Activity and Health, School of Human Movement and Nutrition Sciences, The University of Queensland, Brisbane, Queensland, Australia; ${ }^{2}$ Caring Futures Institute and SHAPE Research Centre, Exercise Science and Clinical Exercise Physiology, College of Nursing and Health Sciences, Flinders University, Adelaide, South Australia, Australia; ${ }^{3}$ Recreation, Exercise, and Sport Science Department, Western State Colorado University, Gunnison, Colorado, USA;

${ }^{4}$ Immunopathology Group, Mater Research Institute - The University of Queensland, Translational Research Institute, Brisbane, Queensland, Australia; ${ }^{5}$ Australian Infectious Disease Research Centre, University of Queensland, Brisbane, Queensland, Australia; ${ }^{6}$ Faculty of Medicine, Dentistry and Health Sciences, The University of Melbourne, Melbourne, Victoria, Australia; ${ }^{7}$ K.G. Jebsen Center of Exercise in Medicine, Department of Circulation and Medical Imaging, Faculty of Medicine, Norwegian University of Science and Technology, Trondheim, Sor Trondelag, Norway

Correspondence: Joyce S Ramos Email joyce.ramos@flinders.edu.au
Introduction: IL-22 may have a role in the alleviation of the metabolic syndrome (MetS) via protection of pancreatic beta and endothelial cells from oxidative and lipid-induced damage. We aimed to investigate the effects of moderate-intensity continuous training (MICT) and different volumes of high-intensity interval training (HIT) on changes in circulating IL-22.

Methods: This was a sub-study of the "Exercise in the prevention of Metabolic Syndrome" (EX-MET) a multi-center, randomized trial. This study used data collected at the Brisbane site. Thirty-nine individuals with MetS were randomized to one of three 16-wk interventions: 1) MICT ( $\mathrm{n}=10,30 \mathrm{~min}$ at $60-70 \%$ HR peak, $5 \mathrm{x} / \mathrm{wk})$; 2) $4 \mathrm{HIIT}(\mathrm{n}=13,4 \mathrm{x} 4 \mathrm{~min}$ at $85-95 \% \mathrm{HR}$ peak, interspersed with $3 \mathrm{~min}$ of active recovery at $50-70 \%$ HR peak, $3 \mathrm{x} / \mathrm{wk}$ ); or 3 ) $1 \mathrm{HIIT}$ $(\mathrm{n}=16,1 \mathrm{x} 4 \mathrm{~min}$ at $85-95 \%$ HR peak, 3x/wk). Serum IL-22 concentration was measured following a 12-hr fast via an enzyme linked immunosorbent assay, before and after the intervention. MetS severity, insulin resistance (IR), visceral adipose tissue (VAT), and cardiorespiratory fitness (CRF) were also measured via MetS z-score, HOMA-IR, dualenergy X-ray absorptiometry, and indirect calorimetry (maximal exercise test), respectively. Results: The median (IQR) IL-22\% changes from pre- to post-intervention in the MICT, 4HIIT, and 1HIIT groups were $-17 \%(-43.0 \%$ to $31.3 \%),+16.5 \%(-18.9 \%$ to $154.9 \%)$, and $+15.9 \%$ ( $-28.7 \%$ to $46.1 \%$ ), respectively. Although there were no significant between-group differences in IL-22 concentration change, there was a medium-to-large group $\times$ time interaction effect $\left[\mathrm{F}(2,35)=2.08, \mathrm{p}=0.14, \eta^{2}=0.14\right]$.

Conclusion: Although there was no statistically significant between-group difference in IL-22 change, the study suggests that different exercise intensities may have opposing effects on IL-22 concentration in individuals with MetS.

Keywords: high-intensity interval training, IL-22, inflammation, cardiovascular disease, metabolic syndrome, cytokine

\section{Introduction}

The immune system has been recognized to be involved in the regulation of metabolic homeostasis. ${ }^{1}$ In particular, interleukin-22 (IL-22), which is part of the IL-10 family of cytokines known to protect organs and tissues from damage caused by infections and inflammatory responses, ${ }^{2}$ has been put forth to play a role in the alleviation of metabolic syndrome (MetS). Two recent studies indicated that IL-22 treatment restores mucosal immunity in diabetes and alleviates metabolic disease, resulting in improved glycemic control. ${ }^{3,4}$ Specifically, it was demonstrated that IL-22 protected 
against oxidative stress from pancreatic beta cells; ${ }^{3}$ and glucose and lipid-induced injury from human endothelial cells. ${ }^{4}$ These are important findings given that: 1) pancreatic beta-cell dysfunction is associated with increased severity of MetS; ${ }^{5}$ and 2) MetS patients with endothelial dysfunction are three times more at risk of cardiovascular events when compared to individuals with only one of either conditions (MetS or endothelial dysfunction). ${ }^{6}$

The role of IL-22 in human health was further highlighted in a study which showed that healthy centenarians have higher circulating IL-22 compared to sex-matched controls aged 60-95 years. ${ }^{7}$ In contrast, it has also been suggested that IL-22 may be detrimental to health, given that circulating IL-22 has been shown to be elevated in people with type 2 diabetes (T2DM), coronary artery disease (CAD), and comorbidity patients (T2DM and CAD). ${ }^{4}$ However, it could be argued that the elevated circulating IL-22 found in these clinical populations may be due to higher IL-22 required to protect factors (ie, pancreatic beta-cell and endothelial dysfunction) associated with these conditions.

Moderate-intensity aerobic training in combination with progressive resistance training significantly reduces IL-22 release in polyclonally activated $\mathrm{T}$ cells following 12-weeks of training, ${ }^{8}$ however, the effect of different exercise intensities on this biomarker has yet to be explored. Although it should be noted that our group showed that neither 12-weeks of MICT nor highintensity interval training (HIIT), which consists of bouts of high-intensity exercise interspersed with recovery periods, had an impact on IL-10. ${ }^{9}$ Nevertheless, HIIT has been shown to improve vascular function ${ }^{10}$ and pancreatic beta-cell function ${ }^{11}$ more than moderateintensity continuous training (MICT), which are both factors previously shown to be protected by IL- $22^{3,4}$ and to be positively associated with cardiorespiratory fitness (CRF). ${ }^{12-14}$ Rana et $\mathrm{al}^{15}$ specifically showed that CRF blunts the inflammatory response in individuals with MetS, wherein those at the highest tertile of CRF revealed no increase in the level of white blood cell count (a marker of inflammation) with increasing MetS risk factors. HIIT has also grown in popularity in recent years due to its ability to improve CRF by twice as much as MICT with the same time commitment. ${ }^{16}$ More recently, lower volumes of HIIT have shown efficacy in providing similar CRF improvements relative to MICT with much shorter exercise times. ${ }^{17}$
The aim of this study was to investigate the impact of MICT and different volumes of HIIT on circulating IL-22 in individuals with MetS. We hypothesized that HIIT, regardless of the exercise volume, would improve IL-22 concentrations more than MICT.

\section{Methods}

\section{Participants and Study Design}

Ninety-nine individuals diagnosed with MetS according to the International Diabetes Federation criteria ${ }^{18}$ were recruited from January 2013 to August 2015 as part of the "Exercise in prevention of Metabolic Syndrome" (EX-MET) multi-center international trial (registered at ClinicalTrials.gov, registration number: NCT01676870). The purpose of the EX-MET trial is to investigate the effect of MICT and varying volumes of HIIT in reducing MetS risk factors. The present sub-study reports the change in IL-22 that was only measured at our local site (Brisbane, Australia). This is an exploratory study due to the availability of samples and emerging evidence on the role of IL-22 on cardiometabolic health. ${ }^{3,4}$ Figure 1 presents the participant flow within the study. Sample size was calculated using an anticipated effect size of mean difference in IL-22 change of 0.42 (power $=0.80$, alpha $=0.05$ for 2-tailed test) between HIIT and MICT groups. This was based on a previous study showing a similar mean difference in IL-10 change between HIIT and MICT in a similar population included in the present study (patients with type 2 diabetes) ${ }^{9}$ since IL-22 is part of the IL-10 cytokine family. ${ }^{2}$

Thirty-nine eligible participants were randomized (stratified by age, sex, and center) into one of the 16-week exercise interventions 1) MICT ( $\mathrm{n}=10$, [males, $\mathrm{n}=5$; females $=n=5])$; 2) 4HIIT ( $\mathrm{n}=13$, [males, $\mathrm{n}=9$; females, $\mathrm{n}=4]$ ); or 3) 1 HIIT ( $n=16$, [males, $n=11$; females, $n=5])$. Randomization procedures were performed by the primary investigators of the multi-center trial from the Norwegian University of Science and Technology (NTNU, Trondheim, Norway), using random permuted blocks. Details of eligible participants were entered into an online system to obtain the training group allocation. This study was approved by the Medical Research Ethics Committee, The University of Queensland, Brisbane, Australia (ethics approval number 2012000617) and conducted in accordance with the Declaration of Helsinki.

Participants were recruited via referrals from medical practitioners at the Princess Alexandra Hospital, and 


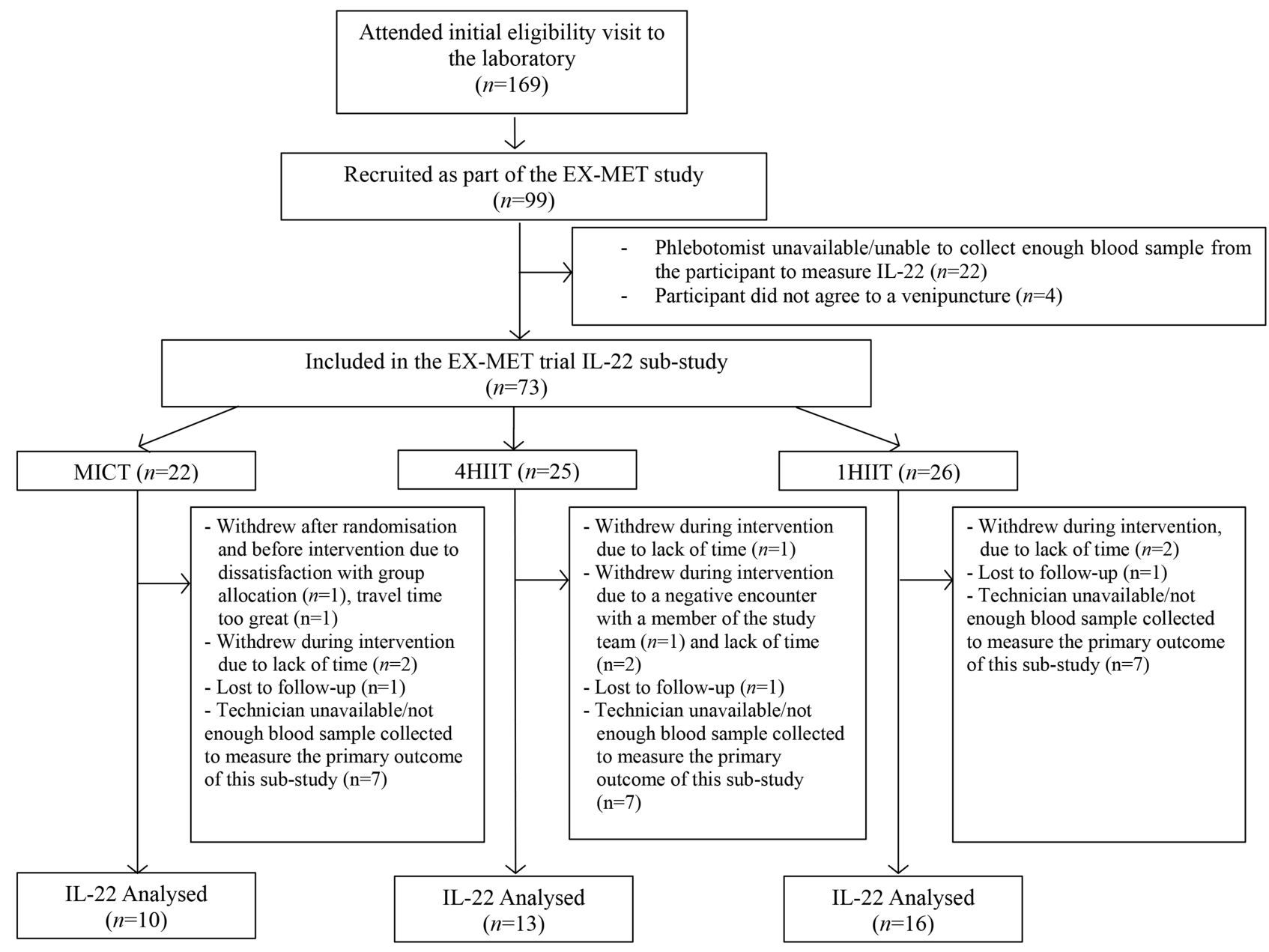

Figure I Flowchart for IL-22 sub-study. MICT, moderate-intensity continuous training; 4HIIT, $4 \times 4$ min high-intensity interval training; I 4 min high-intensity interval training.

advertising through television news, posters, newspapers and flyers posted around the University and local healthcare centers in Brisbane, Australia. A website was also created to serve as a recruitment link in several social media platforms (ie, Facebook and the University's online magazine). Oral and written informed consent were acquired from all participants before study inclusion. Exclusion criteria of the study were unstable angina, recent myocardial infarction (4 weeks), severe valvular heart disease, uncompensated heart failure, pulmonary disease, uncontrolled hypertension, kidney failure, and cardiomyopathy.

Participants' eligibility was determined through the following assessments conducted at a 12-hour fasted state: 1) fasting lipid profile and glucose level; 2) brachial systolic BP (SBP) and diastolic BP (DBP); and 3) anthropometric measures (weight, height, waist circumference [WC] and hip circumference $[\mathrm{HC}]$ ). Detailed description of these procedures has been previously reported. ${ }^{19}$
Before and after completing the 16-week exercise interventions, a series of tests were conducted to measure the primary and secondary outcomes of this sub-study: IL22 and HsCRP; MetS severity; insulin resistance (IR); visceral adipose tissue and other body composition indices; and cardiorespiratory fitness (CRF). All secondary outcome measures of this sub-study have been previously reported. ${ }^{20}$ Participants were instructed to refrain from consumption of caffeine and alcohol for at least 24 hours prior to testing, and participation in strenuous physical activities for at least 48 hours before testing. The following tests to assess the aforementioned outcome measures were performed sequentially at both time points (baseline and post-16-week exercise intervention): blood samples were obtained for later assessment of various biomarkers (IL-22, HsCRP, IR), blood pressure assessment, dualenergy X-ray absorptiometry and other anthropometric assessments (body composition indices), and maximal 
exercise test (CRF). At post-16 week intervention, the assessments were conducted at 48 hours following the last exercise session.

\section{Circulating Interleukin-22 and High-Sensitivity C-Reactive Protein}

Following a 12-hour overnight fast, blood samples were drawn from the participants' antecubital vein. Serum samples were derived from whole blood collected into blood tubes without anticoagulants. These tubes were left at room temperature for 30 minutes prior to centrifugation at $2500 \mathrm{rpm}$ for $10 \mathrm{~min}$ at $4^{\circ} \mathrm{C}$. Serum aliquots were stored at $-80^{\circ} \mathrm{C}$ for later analysis of IL-22 and HsCRP. Concentration of human IL-22 in serum samples was determined using the ELISA MAX ${ }^{\mathrm{TM}}$ Deluxe Set (Biolegend, 434504) using the manufacturer's instructions. In brief, the plates were coated with the capture antibody for 24 hours at $4^{\circ} \mathrm{C}$. Subsequently, plates were washed with PBS- $0.05 \%$ Tween 20 and non-specific binding was blocked using $1 \%$ BSA in PBS ( $\mathrm{pH}$ 7.4) for 2 hours at room temperature, shaking at $500 \mathrm{rpm}$. Plates were then coated with serum samples (1:100 dilution) for up to 3 hours, before being detected using the detection antibody, Avidin-HRP and TMB substrate provided. Absorbance was read at $570 \mathrm{~nm}$ and $450 \mathrm{~nm}$ within $15 \mathrm{~min}$ (data presented as Absorbance 570 nm- $450 \mathrm{~nm}$ against the standard curve for hIL-22 provided). Samples were measured in duplicate, with an intra- and inter-assay co-efficient of variation of $1.9 \%$ and $13 \%$, respectively. HsCRP was also measured via ELISA kit according to the manufacturer's instructions (K-ASSAY High-Sensitive C-Reactive Protein kit KAI-160, Kamiya Biomedical).

\section{Metabolic Syndrome Severity and Insulin Resistance}

Sex-specific MetS z-score was calculated using the following equations ${ }^{21}$ to represent the severity of the metabolic syndrome: (1) MetS z-score men $[(40$ - HDL-C)/8.9] $+[(\mathrm{TG}-150 / 69)]+[(\mathrm{FG}-100) / 17.8]+[(\mathrm{WC}-102) / 11.5]$ $+[(\mathrm{MAP}-100) / 10.1] ;$ (2) MetS z-score ${ }_{\mathrm{women}}=[(50-$ HDL-C $) / 14.5]+[(\mathrm{TG}-150 / 69)]+[(\mathrm{FG}-100) / 17.8]+$ $[(\mathrm{WC}-88) / 12.5]+[(\mathrm{MAP}-100) / 10.1]$, where TG is triglycerides, MAP is mean arterial pressure, and FG is fasting glucose. Insulin resistance was also determined via homeostasis model assessment-2 (HOMA-2) calculator version $2.2 .^{22}$

\section{Visceral Adipose Tissue and Other Body Composition Indices}

Body composition (lean body mass, body fat $\%$, and trunk fat \%) was assessed by dual-energyX-ray absorptiometry (DEXA), as previously described. ${ }^{19}$ Visceral Adipose Tissue (VAT) cross sectional mass was quantified utilising the Advanced Body Composition ${ }^{\mathrm{TM}}$ with InnerCore ${ }^{\mathrm{TM}}$ Visceral Fat Assessment software (Version 4.5.3). Measurements were post-processed with manual editing when appropriate as per manufacturer guidelines. ${ }^{23}$

\section{Cardiorespiratory Fitness}

Participant's CRF was determined as the peak oxygen consumed $\left(\dot{\mathrm{VO}}_{2}\right.$ peak, highest 15 -sec time averaged $\left.\dot{\mathrm{VO}}_{2}\right)$ during a graded maximal exercise test, measured via indirect calorimetry using a MetaMax II (Cortex, Leipzig, Germany) or Parvo Medica TrueOne 2400 system (ParvoMedics Inc., Sandy, Utah, USA). Further details of the maximal exercise test have been described previously. ${ }^{19}$

\section{Exercise Training Protocol}

The frequency of exercise sessions prescribed for the MICT and both HIIT training groups were five and three times per week, respectively, to align with current guidelines for frequency of moderate- and vigorous-intensity exercise from the American College of Sports Medicine. ${ }^{24}$ All exercise groups were required to attend two supervised sessions with an exercise physiologist at The University of Queensland exercise laboratory, and the remaining prescribed training sessions were completed in an unsupervised environment. Supervised sessions were performed either on a treadmill or cycle ergometer, depending on the participants' preference or orthopedic limitations. Unsupervised sessions consisted of outdoor/ indoor pursuits involving large muscle groups, including swimming, walking, running, and rowing. The heart rate and rate of perceived exertion (RPE) during all exercise sessions were monitored and recorded using a heart rate monitor (Polar Electro, Kempele, Finland) and the Borg 6-20 scale, respectively. A training log was also provided to all participants to record HR and RPE during the unsupervised sessions. Figure 2 portrays the schematic for all exercise interventions. Participants in the MICT group exercised continuously for 30 -min each session at $60-70 \%$ of peak heart rate (HR peak) or RPE of 11-13 on the Borg scale ${ }^{25}$ (Figure 2A). Each HIIT session was preceded with a 10-min warm-up and ended with a 3-min 


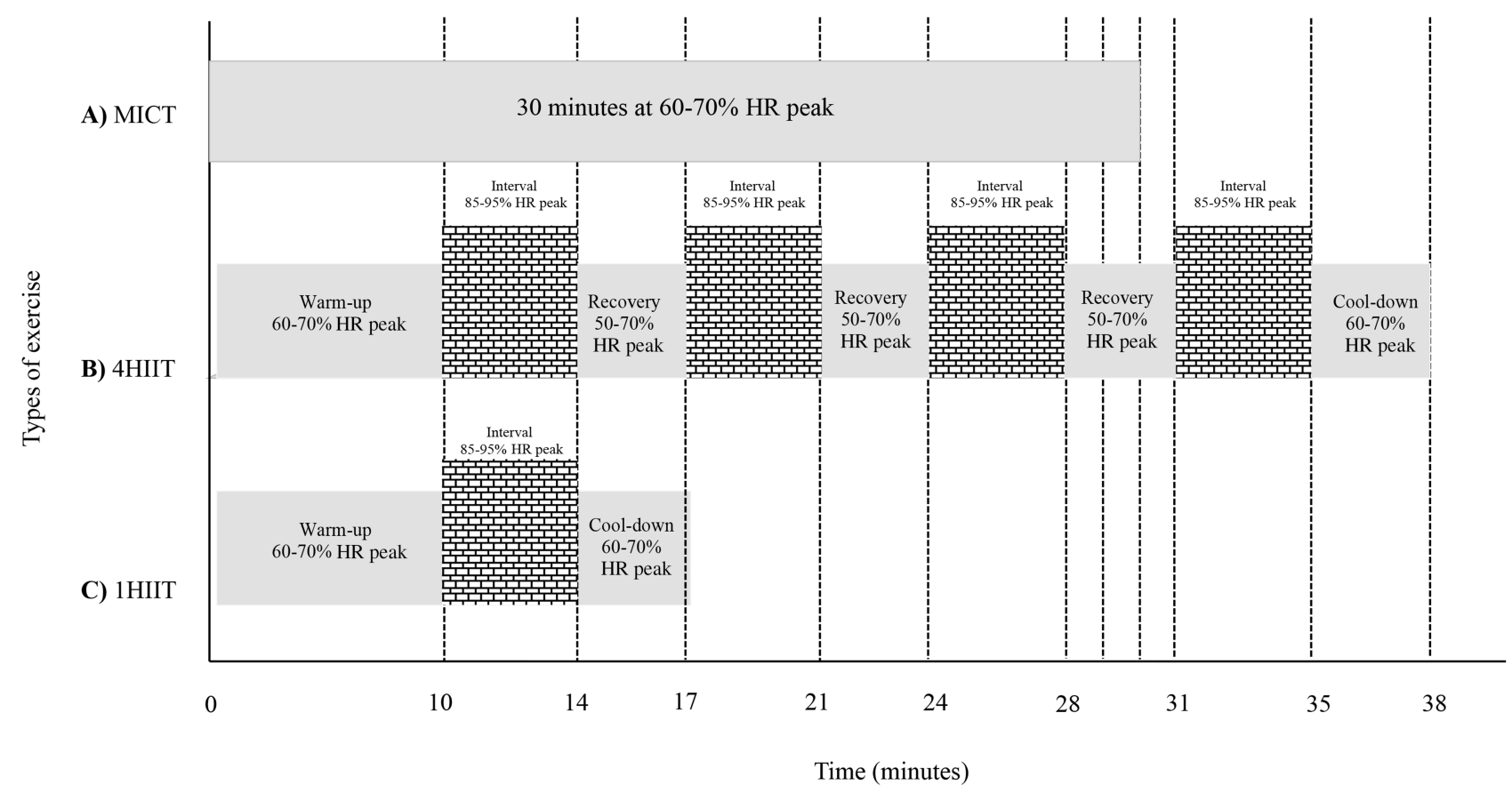

Figure 2 Schematic representation of MICT (A), 4HIIT (B) and IHIIT (C).

cool-down, with a total exercise time of 38 and $17 \mathrm{~min}$ per session for the 4HIIT and 1HIIT, respectively. The 4HIIT protocol incorporated four bouts of 4 min intervals at $85-95 \%$ HR peak/RPE of $15-17$ on Borg scale, interspersed with 3 min of active recovery at 50-70\% HR peak (Figure 2B). The 1HIIT intervention had a similar protocol, but with only one 4 min interval bout at $85-95 \%$ HR peak/RPE of 15-17 on Borg scale (Figure 2C).

\section{Statistical Analysis}

Statistical analyses were performed using SPSS version 22.0 software package (IBM, New York, NY). The Shapiro-Wilk test was used to examine whether parametric tests were appropriate to analyze the data. If the assumption for normality was still violated after log transformation of data, a non-parametric test was applied. Oneway analysis of variance (ANOVA) or Kruskal Wallis, and Chi-square tests were used to compare between-group difference in baseline demographics, medication usage, and exercise adherence. Analysis of covariance (ANCOVA) was used to examine group X time interaction effects, with the baseline value considered as the covariate and the change from pre- to post-intervention value as the dependent variable. Group X time interaction effect sizes $\left(\eta^{2}\right)$ were calculated and interpreted as: "small" effect (0.01); "small-to-medium" effect (0.01-0.10); "mediumto-large" $(0.10-0.25){ }^{26}$
Within-group differences in continuous variables were analyzed via a paired $t$-test or Wilcoxon test. Within-group effect size of the training interventions was calculated using "Cohen's d" (for normally distributed data) or "r" (for non-normally distributed data) effect size which was interpreted as: i) Cohen's d effect size, "small" effect (0.20); small-to-medium (0.20-0.50); and medium-to-large effect (0.50-0.80); ii) r effect size, "small" effect (0.10); small-to-medium (0.10-0.30); and medium-to-large effect $(0.30-0.50) .{ }^{27}$ Mixed linear model regression analysis was also performed to investigate the measures associated with the change in circulating IL-22 within each exercise intervention (4HIIT, 1HIIT, and MICT), with a model that takes into account subjects within a repeated measures design, where IL-22 raw values are the dependent variable and MetS severity, insulin resistance, CRF, and VAT are the individual covariates entered within each training intervention group Continuous variables are reported as mean change \pm standard deviation (SD), or percent or absolute median change (interquartile range $[\mathrm{IQR}]$ ), and categorical variables as frequencies. A $\mathrm{p}$ value $\leq 0.05$ was considered statistically significant.

\section{Results}

Thirty-nine out of the ninety-nine participants recruited as part of the EX-MET trial from January 2013 to 
August 2015, had data for circulating IL-22 before and after the interventions (Figure 1). At baseline, there were no significant group differences in demographics and medication intake (Table 1). Participants in the MICT, 4HIIT, and 1HIIT groups completed $90 \pm 12 \%, 89 \pm 8 \%$, and $93 \pm 7 \%$ of the prescribed sessions (between groups, $\mathrm{p}=0.40$ ), respectively.

\section{Circulating IL-22 and HsCRP}

Table 2 presents changes in circulating IL-22 and HsCRP from pre- to post-intervention. The HIIT groups increased IL-22 and MICT group decreased IL-22. Although there was no significant between-group difference in IL-22 concentration change, there was a medium-to-large group $\mathrm{X}$ time interaction effect on this cytokine $[\mathrm{F}(2,35)=2.08$, $\left.p=0.14, \eta^{2}=0.14\right]$. The same pattern of HsCRP change was also evident following the training interventions, where the HIIT groups increased HsCRP, whilst the MICT group decreased HsCRP, with a small-to-medium group $\mathrm{X}$ time interaction effect $\left[\mathrm{F}(2,37)=1.47, \mathrm{p}=0.24, \eta^{2}=0.07\right]$.

\section{MetS Z-Score and Insulin Resistance}

Table 2 also presents the MetS z-scores and individual MetS components following the exercise interventions. MetS z-score decreased following all exercise programs (MICT, $-0.5 \pm 1.8$; 4HIIT, $-0.5 \pm 0.9$; 1 HIIT, $-1.3 \pm 1.8$ ), with no significant difference between groups after adjusting for baseline values $\left[\mathrm{F}(2,38)=1.21, \mathrm{p}=0.31, \eta^{2}=0.06\right]$. There was a greater magnitude of insulin resistance (HOMA-

Table I Participants' Characteristics

\begin{tabular}{|l|l|l|l|l|}
\hline Variables & $\begin{array}{l}\text { MICT } \\
(\mathbf{n}=\mathbf{I 0})\end{array}$ & $\begin{array}{l}\text { 4HIIT } \\
(\mathbf{n}=\mathbf{1 3})\end{array}$ & $\begin{array}{l}\text { IHIIT } \\
(\mathbf{n}=\mathbf{1 6})\end{array}$ & P-value \\
\hline Demographics & & & & \\
Age, years, mean \pm SD & $54 \pm 9$ & $54 \pm 10$ & $57 \pm 8$ & 0.47 \\
Male sex, \% & 50 & 69 & 69 & 0.56 \\
Type 2 diabetes, \% & 20 & 62 & 56 & 0.10 \\
Hypertension, \% & 60 & 85 & 75 & 0.41 \\
\hline Medications & & & & \\
ACEls, \% & 30 & 46 & 56 & 0.43 \\
Calcium Antagonist, \% & 0 & 23 & 13 & 0.26 \\
Beta-blocker, \% & 20 & 0 & 19 & 0.24 \\
Statin, \% & 20 & 46 & 63 & 0.11 \\
Aspirin, \% & 20 & 23 & 19 & 0.96 \\
Metformin,\% & 10 & 38 & 38 & 0.26 \\
\hline
\end{tabular}

Abbreviations: MICT, moderate-intensity continuous training; 4HIIT, 4xX4min high-intensity interval training; IHIIT, IX4min high-intensity interval training; ACEls, angiotensin-converting enzyme inhibitors.
IR) reduction following MICT (MICT, $-2.1 \pm 2.7$ ) relative to the HIIT groups (4HIIT, $-0.8 \pm 1.4 ; 1 \mathrm{HIIT},-1.0 \pm 2.0$ ), but with no significant difference between groups $[\mathrm{F}(2,38)$ $\left.=0.92, \mathrm{p}=0.41, \eta^{2}=0.05\right]$.

\section{Cardiorespiratory Fitness}

The 4HIIT intervention significantly improved CRF $(+5.7$ $\pm 4.7 \mathrm{~mL} / \mathrm{kg} / \mathrm{min})$ more than MICT $(+2.7 \pm 1.9 \mathrm{~mL} / \mathrm{kg} / \mathrm{min})$ and 1 HIIT $(+2.9 \pm 2.2 \mathrm{~mL} / \mathrm{kg} / \mathrm{min})$ training groups $[\mathrm{F}(2,38)$ $\left.=3.8, \mathrm{p}=0.03, \eta^{2}=0.18\right]$.

\section{VAT and Other Body Composition Indices}

Table 2 shows similar reductions in VAT (MICT, -47.2 $\pm 152.6 \mathrm{~g}$; 4HIIT, $-68.2 \pm 106.0 \mathrm{~g}$; $1 \mathrm{HIIT},-44.9 \pm 102.7 \mathrm{~g})$ and negligible changes in all other body fat indices (total body fat $\%$, android fat $\%$, and gynoid fat $\%$ ) and lean mass following the training interventions. After exercise training both HIIT groups showed a greater magnitude of weight reduction when compared to MICT: (4HIIT, $-2.7 \pm 5.9 \mathrm{~kg}$; 1HIIT, $-2.3 \pm 3.9 \mathrm{~kg}$ ) vs (MICT, $-0.4 \pm 4.5 \mathrm{~kg}$ ).

\section{Mixed Linear Model Regression Analysis}

A mixed linear model regression analysis was used to further examine the associations between changes in MetS severity, insulin resistance, CRF, and VAT and the change in IL-22 concentrations following MICT, 4HIIT, and 1HIIT. Our analyses showed that the change in IL-22 concentration was significantly associated with the change in CRF following 1HIT $(\mathrm{p}=0.04)$. IL-22 change was not significantly associated with any other changes in the aforementioned factors following all exercise interventions.

\section{Discussion}

This is the first study to investigate the impact of different exercise intensities on circulating IL-22 in patients with MetS. Although there was no statistically significant difference in IL-22 change between exercise training groups, our results suggest that different exercise intensities may have opposite effects on circulating IL-22 levels.

Both HIIT groups increased the magnitude of IL-22 following the 16-week intervention, whilst the MICT group decreased the magnitude of this anti-inflammatory cytokine. It should be highlighted however that despite the differential IL-22 change between exercise intensities, a clinical reduction in MetS severity was evident following all training interventions. Moreover, a parallel favorable 


\begin{tabular}{|c|c|c|c|c|c|c|c|c|c|c|c|}
\hline \multirow{2}{*}{ 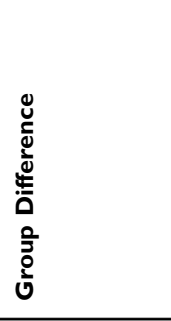 } & 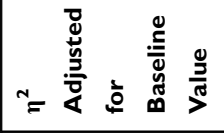 & $\frac{t}{0}$ & 옹 & $\stackrel{\circ}{\circ}$ & & $\bar{o}$ & $\frac{\Lambda}{0}$ & $\bar{o}$ & ర్ & ¿̊. & $\bar{o}$ \\
\hline & 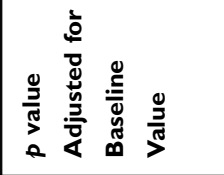 & $\frac{\pi}{0}$ & 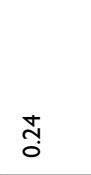 & $\bar{m}$ & & $\stackrel{\substack{0 \\
0}}{0}$ & $\stackrel{m}{0}$ & $\underset{\infty}{\infty}$ & 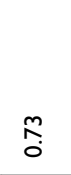 & $\bar{ָ}$ & $\stackrel{+}{\hat{O}}$ \\
\hline 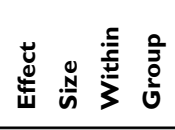 & 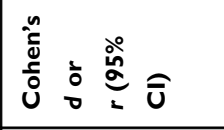 & రి & ợ & ơ & & ઼ָ & 音 & $\frac{0}{0}$ & $\frac{n}{0}$ & క్ర & $\stackrel{+}{0}$ \\
\hline \multirow{2}{*}{$\begin{array}{l}\frac{6}{11} \\
\qquad \\
\underline{\underline{I}}\end{array}$} & 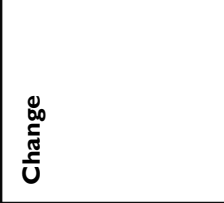 & 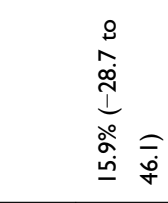 & $\begin{array}{l}\pi \\
0 \\
8 \\
\vdots \\
1 \\
\stackrel{0}{0} \\
m\end{array}$ & $\frac{\infty}{+1}$ & & $\underset{\substack{0 \\
+1}}{\stackrel{+}{+}}$ & \begin{tabular}{l}
\multirow{2}{0}{} \\
+1 \\
0 \\
0
\end{tabular} & $\begin{array}{l}0 \\
0 \\
0 \\
0 \\
0 \\
0 \\
0 \\
0 \\
0 \\
0 \\
0\end{array}$ & $\underbrace{+}_{i}$ & $\begin{array}{l}9 \\
\text { +1 }\end{array}$ & $\begin{array}{l}0 \\
0 \\
a \\
i \\
0\end{array}$ \\
\hline & 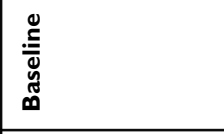 & 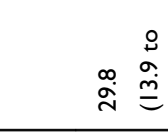 & $\stackrel{\stackrel{0}{\infty}}{\stackrel{\infty}{a}} \frac{\sigma}{\rho}$ & $\stackrel{\sim}{\sim}$ & & 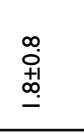 & $\begin{array}{l}\stackrel{+}{0} \\
\stackrel{+}{+} \\
\stackrel{+}{-}\end{array}$ & 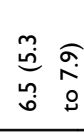 & $\frac{m}{+1}$ & $\frac{m}{\stackrel{m}{+1}}$ & $\underset{+}{+}$ \\
\hline 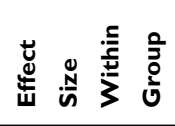 & 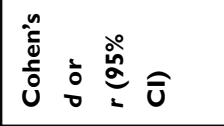 & ợ & 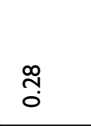 & สุ & & o̊ & $\overline{0}$ & ঃ̊ & $\frac{\overline{0}}{0}$ & ণ్రి & $\hat{\circ}$ \\
\hline \multirow[b]{2}{*}{ 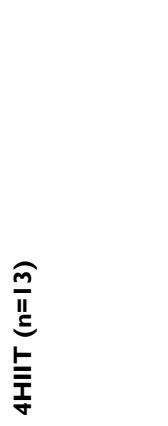 } & ర్ & 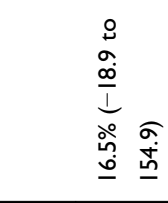 & 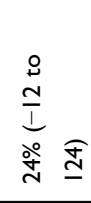 & 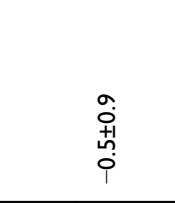 & & 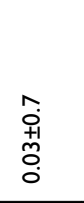 & 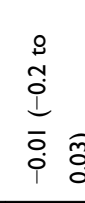 & $\begin{array}{l}0 \\
0 \\
0 \\
0 \\
0 \\
0 \\
0 \\
0 \\
i\end{array}$ & $\stackrel{\sim}{\uparrow}$ & $\stackrel{\circ}{ \pm 1}$ & $\stackrel{\text { 辛 }}{\text { p }}$ \\
\hline & 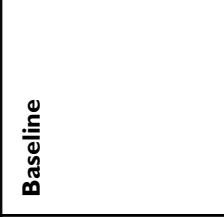 & 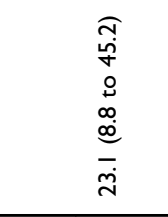 & 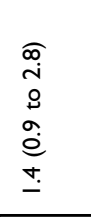 & $\hat{i}$ & & $\begin{array}{l}\infty \\
\stackrel{\infty}{+} \\
\stackrel{i}{i}\end{array}$ & 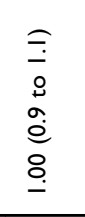 & 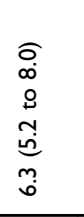 & 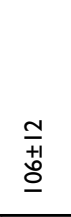 & $\begin{array}{l}\stackrel{\circ}{+1} \\
\stackrel{+}{0}\end{array}$ & 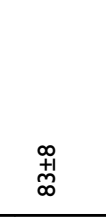 \\
\hline 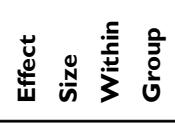 & 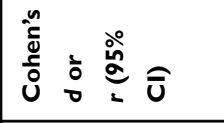 & $\frac{n}{0}$ & $\stackrel{\substack{\infty \\
0}}{0}$ & สุ & & o̊. & ò & $\frac{0}{0}$ & $\stackrel{\circ}{\circ}$ & $\tilde{\tilde{o}}$ & ? \\
\hline \multirow{3}{*}{$\begin{array}{l}\frac{0}{11} \\
\stackrel{5}{5} \\
\frac{u}{\Sigma}\end{array}$} & 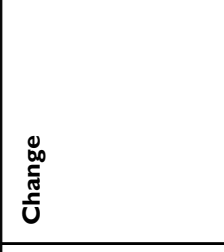 & $\begin{array}{l}\widehat{m} \\
\frac{m}{m} \\
\dot{0} \\
\stackrel{y}{ \pm} \\
\stackrel{0}{\circ} \\
\frac{1}{1}\end{array}$ & 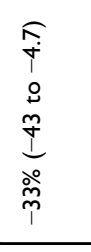 & 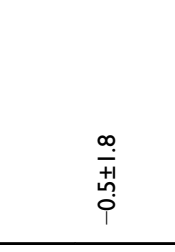 & & $\begin{array}{l}\text { f. } \\
0 \\
0 \\
0 \\
0 \\
0 \\
i \\
0 \\
0 \\
0\end{array}$ & 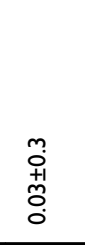 & $\begin{array}{l}\widehat{f} \\
0 \\
0 \\
0 \\
0 \\
0 \\
\text { I } \\
\text { ñ } \\
i\end{array}$ & $\frac{2}{1}$ & 辛 & 卢 \\
\hline & 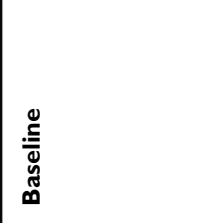 & 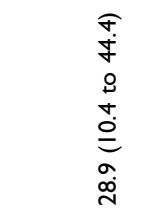 & $\begin{array}{l}\widehat{\bar{m}} \\
\stackrel{0}{0} \\
\infty \\
\stackrel{\infty}{0} \\
\infty \\
\stackrel{\infty}{.}\end{array}$ & $\stackrel{\infty}{-}$ & & 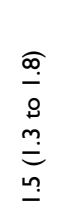 & 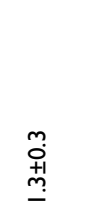 & 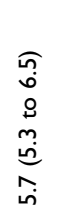 & $\begin{array}{l}\stackrel{n}{1} \\
\stackrel{0}{0} \\
\underline{0}\end{array}$ & $\underset{m}{\frac{m}{0}}$ & $\begin{array}{l}\circ \\
\stackrel{\circ}{+1} \\
\infty\end{array}$ \\
\hline & & 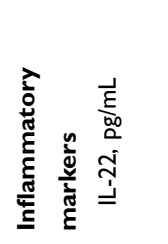 & 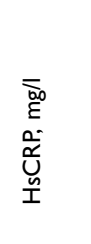 & 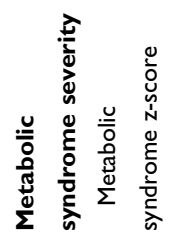 & 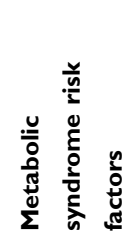 & 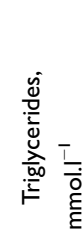 & 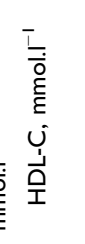 & 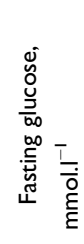 & $\sum_{0}^{\frac{4}{n}}$ & 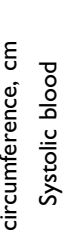 & 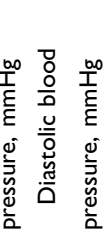 \\
\hline
\end{tabular}




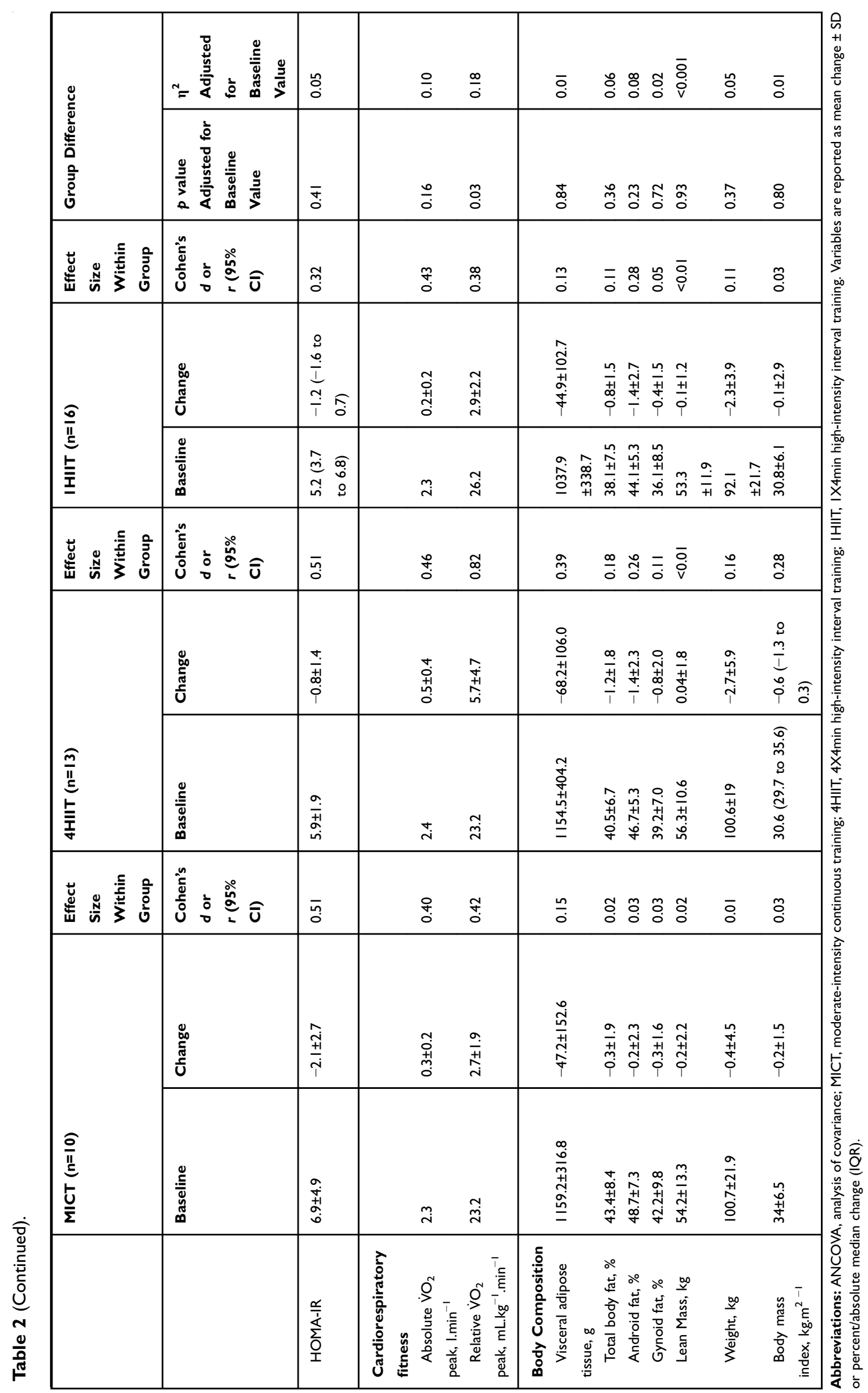


change in insulin resistance, VAT, and CRF, all known factors to either play a central role in the emergence of $\mathrm{MetS}^{28}$ or to be an antidote against individual MetS components, ${ }^{29}$ were also evident following all exercise regimens. It should be noted, however, that our results showed that a change in IL-22 concentration was only associated with a change in CRF following 1HIIT. IL-22 change within group was not associated with changes in MetS severity, insulin resistance, VAT following all exercise interventions, perhaps due to the low sample size within each group.

Consistent with the present study, Alvarenga-Filho et $\mathrm{al}^{8}$ also found a decrease in circulating IL-22 concentrations following a 12 -week MICT exercise program. It is plausible that the circulating IL-22 reduction following MICT may in part be attributable to the direct capacity of this exercise intensity to improve factors known to aggravate IL-22-protected components (ie, endothelial and pancreatic beta cells) ${ }^{10,30}$ associated with the severity of MetS, thereby reducing the need for further IL-22 immunity. Indeed, MICT has been shown to be a potent stimulus towards improving endothelial ${ }^{10}$ and pancreatic beta-cell function. ${ }^{30}$ Interestingly, the present study demonstrated a greater magnitude of insulin resistance reduction following MICT compared to the HIIT groups. Thus, given that insulin resistance has been proposed to be a major culprit underpinning endothelial ${ }^{31}$ and pancreatic beta cells ${ }^{32}$ dysfunction, it could be speculated that the observed degree of insulin resistance reduction could have led to a simultaneous decrease in IL-22 following MICT. Further studies are warranted to test this hypothesis.

In contrast, both HIIT interventions in the present study increased the magnitude of circulating IL-22, which were concomitant with improved CRF and favorable reductions in MetS severity, VAT, and insulin resistance. This suggests that high-intensity exercise may stimulate IL-22 production, in addition to its capacity to improve MetS factors that are known to be detrimental to IL-22-protected tissues (ie, pancreatic beta cells and endothelial cells). ${ }^{5,6}$ This may plausibly explain the superior ability of HIIT compared to MICT to improve novel CVD risk factors such as vascular function ${ }^{10}$ and pancreatic beta-cell function ${ }^{11}$ demonstrated in previous studies. HIIT has also been reported to elicit superior skeletal muscle mitochondrial function relative to $\mathrm{MICT},{ }^{33}$ perhaps explaining the greater magnitude of CRF increase following HIIT compared to MICT in the present study. Thus, assuming that the mechanism involved in the up-regulation of mitochondrial function is similar across tissues, HIIT-induced IL-22 magnitude increase may be attributed to mitochondrial function improvement of lymphocytes ${ }^{34}$ implicated in the expression and activation of IL-22. ${ }^{35}$

Another interpretation is that the increase in IL-22 magnitude, evident in the HIIT groups, may be partially attributable to a higher IL-22 needed to compensate for an increase in pro-inflammatory cytokines, which are usually evident following strenuous exercise. ${ }^{36}$ Indeed, this scenario is supported by our findings that increased HsCRP concentrations following both HIIT interventions were concomitant to increased IL-22, whereas an opposite pattern of changes in these biomarkers occurred post-MICT. It is plausible that the blood test we conducted 48 hours after the last exercise session could have merely reflected the acute effects of HIIT on pro-inflammatory markers and IL-22 concentrations. It could be speculated that the elevated IL-22 and HsCRP markers may be due to the imposed acute damage on fast-twitch muscle fibers, which were likely to be predominantly recruited during HIIT. However, Zimowska et $\mathrm{al}^{37}$ showed that the repair of slow-twitch muscle fibers, which were likely to be largely involved during MICT in the present study, is accompanied by increased and prolonged pro-inflammation as compared to the regeneration of fast-twitch muscle fibers. It should also be noted that a previous study has demonstrated a reduction in a pro-inflammatory cytokine in parallel with a favorable change in the anti-inflammatory IL-10 cytokine, in which IL-22 is known to be a key member, following HIIT in patients with MetS. ${ }^{38}$ This is further supported by our results, which showed a parallel decrease in VAT following the HIIT interventions, indicating reduced hypoxia-induced necrosis, and thus secretion of pro-inflammatory cytokines. ${ }^{39}$

\section{Limitations}

The main limitation of this study is that we only measured circulating IL-22, limiting the ability to determine possible tissue-specific improvement induced by different exercise treatments. It would have also been more informative to have had included a set of other pro-and anti-inflammatory markers to better explain possible mechanisms of IL-22 changes in response to differential exercise intensities. It should also be noted that there is a wide variability in our IL-22 result, despite appropriate measures taken to reduce this variability during the trial. We suspect that this may have been influenced by the individual variability in cardiometabolic responses to our exercise interventions and/or dietary 
intake. In particular, our previous study showed that fibre intake is independently associated with increased circulating interleukin-22 in individuals with MetS. ${ }^{40}$ Further studies are warranted to elucidate these factors. Finally, our post hoc power analysis showed that this exploratory study is significantly underpowered (0.29).

\section{Conclusion}

The main novel finding was that different exercise intensities have polar effects on circulating IL-22 concentrations. Notably, our study indicated that exercise intensity regardless of the volume may provide more health benefits by increasing circulating IL-22, known to have a protective role in factors associated with MetS. ${ }^{41}$ It should also be noted that we did not find a statistically significant difference in IL-22 change between exercise interventions. A larger clinical trial is required to confirm our findings.

\section{Data Sharing Statement}

No further data will be shared besides what are already included in the manuscript.

\section{Acknowledgments}

Funding for this study was provided by the Norwegian University of Science and Technology and from an unrestricted research grant from The Coca-Cola Company.

\section{Disclosure}

Professor Jeff S. Coombes reports grants from Norwegian University of Science and Technology and The Coca-Cola Company during the conduct of the study. Dr Sumaira Z Hasnain reports a licensed patent: PCT/AU2015/050283. The authors declare no other possible conflicts of interest associated with this work.

\section{References}

1. Sabat R, Wolk K. Deciphering the role of interleukin-22 in metabolic alterations. Cell Biosci. 2015;5(1):68. doi:10.1186/s13578-015-0060-8

2. Ouyang W, Rutz S, Crellin NK, Valdez PA, Hymowitz SG. Regulation and functions of the IL-10 family of cytokines in inflammation and disease. Annu Rev Immunol. 2011;29(1):71-109. doi:10.1146/annurevimmunol-031210-101312

3. Hasnain SZ, Borg DJ, Harcourt BE, et al. Glycemic control in diabetes is restored by therapeutic manipulation of cytokines that regulate beta cell stress. Nat Med. 2014;20(12):1417-1426. doi:10.1038/nm.3705

4. Gong $\mathrm{F}$, Wu J, Zhou $\mathrm{P}$, et al. Interleukin-22 might act as a double-edged sword in type 2 diabetes and coronary artery disease. Mediators Inflamm. 2016;2016:8254797. doi:10.1155/2016/8254797

5. Malin SK, Finnegan S, Fealy CE, Filion J, Rocco MB, Kirwan JP. Beta-Cell dysfunction is associated with metabolic syndrome severity in adults. Metab Syndr Relat Disord. 2014;12(2):79-85. doi:10.1089/ met.2013.0083
6. Suzuki T, Hirata K, Elkind MS, et al. Metabolic syndrome, endothelial dysfunction, and risk of cardiovascular events: the Northern Manhattan Study (NOMAS). Am Heart J. 2008;156(2):405-410. doi:10.1016/j.ahj.2008.02.022

7. Basile G, Paffumi I, D'Angelo AG, et al. Healthy centenarians show high levels of circulating interleukin-22 (IL-22). Arch Gerontol Geriatr. 2012;54(3):459-461. doi:10.1016/j.archger.2011.05.004

8. Alvarenga-Filho H, Sacramento PM, Ferreira TB, et al. Combined exercise training reduces fatigue and modulates the cytokine profile of T-cells from multiple sclerosis patients in response to neuromediators. J Neuroimmunol. 2016;293:91-99. doi:10.1016/j. jneuroim.2016.02.014

9. Mallard AR, Hollekim-Strand SM, Coombes JS, Ingul CB. Exercise intensity, redox homeostasis and inflammation in type 2 diabetes mellitus. J Sci Med Sport. 2017;20(10):893-898. doi:10.1016/j. jsams.2017.03.014

10. Ramos JS, Dalleck LC, Tjonna AE, Beetham KS, Coombes JS. The impact of high-intensity interval training versus moderate-intensity continuous training on vascular function: a systematic review and meta-analysis. Sports Med. 2015;45(5):679-692. doi:10.1007/ s40279-015-0321-z

11. Ramos JS, Dalleck LC, Borrani F, et al. The effect of different volumes of high-intensity interval training on proinsulin in participants with the metabolic syndrome: a randomised trial. Diabetologia. 2016;59(11):2308-2320. doi:10.1007/s00125-016-4064-7

12. Ramos JS, Dalleck LC, Borrani F, Fassett RG, Coombes JS. Cardiorespiratory fitness is positively associated with increased pancreatic beta cell function independent of fatness in individuals with the metabolic syndrome: fitness versus fatness. J Sci Med Sport. 2017;20(1):45-49. doi:10.1016/j.jsams.2016.04.007

13. Buscemi S, Canino B, Batsis JA, et al. Relationships between maximal oxygen uptake and endothelial function in healthy male adults: a preliminary study. Acta Diabetol. 2013;50(2):135-141. doi:10.10 07/s00592-010-0229-x

14. Davison K, Bircher S, Hill A, Coates AM, Howe PR, Buckley JD. Relationships between obesity, cardiorespiratory fitness, and cardiovascular function. J Obes. 2010;2010:191253. doi:10.1155/2010/ 191253

15. Rana JS, Nasir K, Santos RD, et al. Increased level of cardiorespiratory fitness blunts the inflammatory response in metabolic syndrome. Int $J$ Cardiol. 2006;110(2):224-230. doi:10.1016/j. ijcard.2005.08.040

16. Weston KS, Wisloff U, Coombes JS. High-intensity interval training in patients with lifestyle-induced cardiometabolic disease: a systematic review and meta-analysis. Br J Sports Med. 2014;48(16):1227-1234. doi:10.1136/bjsports-2013-092576

17. Tjonna AE, Leinan IM, Bartnes AT, et al. Low- and high-volume of intensive endurance training significantly improves maximal oxygen uptake after 10-weeks of training in healthy men. PLoS One. 2013;8 (5):e65382. doi:10.1371/journal.pone.0065382

18. Alberti KG, Zimmet P, Shaw J. Metabolic syndrome-a new worldwide definition. A Consensus Statement from the International Diabetes Federation. Diabet Med. 2006;23(5):469-480. doi:10.1111/ j.1464-5491.2006.01858.x

19. Ramos JS, Ramos MV, Dalleck LC, et al. Fitness is independently associated with central hemodynamics in metabolic syndrome. Med Sci Sports Exerc. 2016;48(8):1539-1547. doi:10.1249/MSS.0000 000000000916

20. Ramos JS, Dalleck LC, Borrani F, et al. Low-volume high-intensity interval training is sufficient to ameliorate the severity of metabolic syndrome. Metab Syndr Relat Disord. 2017;15(7):319-328. doi:10.1089/met.2017.0042

21. Malin SK, Nightingale J, Choi SE, Chipkin SR, Braun B. Metformin modifies the exercise training effects on risk factors for cardiovascular disease in impaired glucose tolerant adults. Obesity (Silver Spring). 2013;21(1):93-100. doi:10.1002/oby.20235 
22. Diabetes Trials Unit TOCfD. Endocrinology and Metabolism HOMA2 Calculator. Oxford: The University of Oxford; 2011.

23. Kelly T, Wilson K, Ruth C. Estimating visceral fat by dual-energy X-ray absorptiometry. Hologic, Inc.. Bedford MA US. 2010;(10/958 (107)):1-15.

24. Garber CE, Blissmer B, Deschenes MR, et al. American College of Sports Medicine position stand. Quantity and quality of exercise for developing and maintaining cardiorespiratory, musculoskeletal, and neuromotor fitness in apparently healthy adults: guidance for prescribing exercise. Med Sci Sports Exerc. 2011;43(7):1334-1359. doi:10.1249/MSS.0b013e318213fefb

25. Borg GA. Psychophysical bases of perceived exertion. Med Sci Sports Exerc. 1982;14(5):377-381. doi:10.1249/00005768-198205000-00012

26. Vacha-Haase T, Thompson B. How to estimate and interpret various effect sizes. J Couns Psychol. 2004;51(4):473-481. doi:10.1037/ 0022-0167.51.4.473

27. Rosenthal R, Rosnow RL. Essentials of Behavioral Research: Methods and Data Analysis. New York: McGraw-Hill; 1984.

28. Bergman RN, Kim SP, Catalano KJ, et al. Why visceral fat is bad: mechanisms of the metabolic syndrome. Obesity (Silver Spring). 2006;14(Suppl 1):16s-19s. doi:10.1038/oby.2006.277

29. Blair SN, Kampert JB, Kohl HW 3rd, et al. Influences of cardiorespiratory fitness and other precursors on cardiovascular disease and all-cause mortality in men and women. JAMA. 1996;276(3):205-210. doi:10.1001/jama.1996.03540030039029

30. Slentz CA, Tanner CJ, Bateman LA, et al. Effects of exercise training intensity on pancreatic beta-cell function. Diabetes Care. 2009;32 (10):1807-1811. doi:10.2337/dc09-0032

31. Muniyappa R, Sowers JR. Role of insulin resistance in endothelial dysfunction. Rev Endocr Metab Disord. 2013;14(1):5-12. doi:10.1007/ s11154-012-9229-1

32. Cerf ME. Beta cell dysfunction and insulin resistance. Front Endocrinol (Lausanne). 2013;4:37. doi:10.3389/fendo.2013.00037
33. Tjonna AE, Lee SJ, Rognmo O, et al. Aerobic interval training versus continuous moderate exercise as a treatment for the metabolic syndrome: a pilot study. Circulation. 2008;118(4):346-354. doi:10.1161/ CIRCULATIONAHA.108.772822

34. Walker MA, Volpi S, Sims KB, Walter JE, Traggiai E. Powering the immune system: mitochondria in immune function and deficiency. J Immunol Res. 2014;2014:164309. doi:10.1155/2014/164309

35. Dalmas E, Venteclef N, Caer C, et al. T cell-derived IL-22 amplifies IL-1beta-driven inflammation in human adipose tissue: relevance to obesity and type 2 diabetes. Diabetes. 2014;63(6):1966-1977. doi: $10.2337 / \mathrm{db} 13-1511$

36. Pedersen BK. Exercise and cytokines. Immunol Cell Biol. 2000;78 (5):532-535. doi:10.1111/j.1440-1711.2000.t01-11-.x

37. Zimowska M, Kasprzycka P, Bocian K, et al. Inflammatory response during slow- and fast-twitch muscle regeneration. Muscle Nerve. 2017;55(3):400-409. doi:10.1002/mus.25246

38. Steckling FM, Farinha JB, Santos DL, et al. High intensity interval training reduces the levels of serum inflammatory cytokine on women with metabolic syndrome. Exp Clin Endocrinol Diabetes. 2016;124(10):597-601. doi:10.1055/s-0042-111044

39. Pasarica M, Sereda OR, Redman LM, et al. Reduced adipose tissue oxygenation in human obesity: evidence for rarefaction, macrophage chemotaxis, and inflammation without an angiogenic response. Diabetes. 2009;58(3):718-725. doi:10.2337/db08-1098

40. Torquati L, Coombes JS, Murray L, et al. Fibre intake is independently associated with increased circulating interleukin-22 in individuals with metabolic syndrome. Nutrients. 2019;11(4):815. doi:10. 3390/nu11040815

41. Dalmas E, Donath MY. A role for interleukin-22 in the alleviation of metabolic syndrome. Nat Med. 2014;20(12):1379-1381. doi:10.1038/ nm. 3748

Diabetes, Metabolic Syndrome and Obesity: Targets and Therapy

Dovepress

\section{Publish your work in this journal}

Diabetes, Metabolic Syndrome and Obesity: Targets and Therapy is an international, peer-reviewed open-access journal committed to the rapid publication of the latest laboratory and clinical findings in the fields of diabetes, metabolic syndrome and obesity research. Original research, review, case reports, hypothesis formation, expert opinion and commentaries are all considered for publication. The manuscript management system is completely online and includes a very quick and fair peer-review system, which is all easy to use. Visit http://www.dovepress.com/testimonials.php to read real quotes from published authors.

Submit your manuscript here: https://www.dovepress.com/diabetes-metabolic-syndrome-and-obesity-targets-and-therapy-journal 